\title{
UNIVERSIDAD Y HUMANIDADES: LA ENSEÑANZA DE LAS HUMANIDADES EN LA UNIVERSIDAD, UN OBJETO HISTÓRICO DE SABER Y DE PODER
}

\author{
Martín Emilio Camargo Palencia* \\ Profesor, Universidad Pedagógica y Tecnológica de Colombia
}

\section{Resumen}

Universidad y humanidades: la enseñanza de las humanidades en la universidad, un objeto histórico de saber y de poder. La inserción del modelo económico capitalista en la educación ha devenido en una crisis de saberes a nivel mundial. En este sentido una de las mayores preocupaciones de los profesores universitarios de humanidades, ante unos estudiantes cada vez más mediáticos y menos críticos, es la del qué enseñar y cómo enseñar las humanidades con temáticas contextualmente adecuadas y estrategias más pedagógicas. En este artículo consideramos que una respuesta a esta preocupación es menester pensarla a partir de la deconstrucción histórica de la relación universidad y humanidades. En estricto sentido se trataría de una genealogía sobre las condiciones de posibilidad en que las humanidades han sido enseñadas en la universidad. Immanuel Kant, Jean François Lyotard, Jacques Derrida, y más recientemente Martha Nussbaum, son referentes teóricos fundamentales para llevar a cabo tal genealogía.

Palabras clave: Universidad, humanidades, saber y poder.

\begin{abstract}
University and the Humanities: the Teaching of the Humanities at the University, a Historical Object of Knowledge and Power. The introduction of the capitalist

* El presente artículo hace parte de uno de los objetivos del grupo de investigación Filosofía, sociedad y educación, específicamente en su línea de investigación Filosofía de la educación y enseñanza de la filosofía: contribuir a la desmitificación de la enseñanza de las humanidades como un ornamento inútil en un mundo económicamente cada vez más global.
\end{abstract}

Universidad y humanidades - p. 97 - 112 
economic model in education has led to a crisis of global knowledge. With students becoming more media dependent and less critical, one of the major concerns of Humanities professors is that of what to teach and how to teach humanities with contextually appropriate themes and more pedagogical strategies. In response to this concern, we propose the importance of thinking about it from the historical deconstruction of the relationship between the university and humanities. Strictly speaking, this entails a genealogy of the conditions of possibility in which the humanities have been taught at the university. Immanuel Kant, Jean François Lyotard, Jacques Derrida, and more recently Martha Nussbaum, are fundamental theorists when it comes to developing such a genealogy.

Key words: University, humanities, knowledge and power.

La educación tendrá por objeto el pleno desarrollo de la personalidad humana y el fortalecimiento del respeto a los derechos humanos y a las libertades fundamentales; favorecerá la comprensión, la tolerancia y la amistad entre todas las naciones y todos los grupos étnicos o religiosos.

Declaración Universal de los Derechos Humanos, 1948.

\section{Introducción}

La enseñanza de las humanidades atraviesa en la actualidad una fuerte crisis en todos los niveles de la escolaridad a nivel mundial. Dicha crisis puede definirse como el privilegio que en la educación se le está dando a la formación de ciudadanos con mentalidad comercial en detrimento de la formación de ciudadanos críticos, creativos y éticos. Esta situación está estrechamente relacionada con las reglas de producción de bienes y servicios impuestas por un mercado único global del cual dependen las economías internas de los Estados democráticos, pues éstos, urgidos de políticas que les garanticen su competitividad económica dentro de dicho mercado, desechan la enseñanza de las humanidades en beneficio del aprendizaje de otros saberes más directamente relacionados con actividades empresariales. Para Martha 
Nussbaum, ${ }^{1}$ esta crisis, a la que denomina "crisis silenciosa" en razón a que avanza subrepticiamente pero con firmeza política, se expresa en la "erosión" de valores como la libertad y la justicia, esenciales en el sostenimiento de una democracia. Este es el aspecto político de la crisis, que a nuestro juicio es fundamentalmente una cuestión de poder. Evidentemente el saber y el poder no son lo mismo. El primero hace referencia a las formas de enunciación y de visibilidad, las cuales tienen un carácter social e histórico; el segundo es la estrategia que determina las relaciones entre las formas de enunciación y de visibilidad. Así pues, todo saber supone un ejercicio de poder que se efectúa en él, y todo ejercicio de poder supone un determinado saber por el que se efectúa. El saber y el poder, entonces, son complementarios en la diferencia y en toda sociedad en términos históricos. ${ }^{2}$

El otro aspecto es el pedagógico, o la cuestión del saber, es decir, la enseñanza misma de las humanidades en términos del qué se enseña y cómo se enseña. Ambos aspectos han sido tratados suficientemente por maestros, intelectuales, investigadores y filósofos como Kant, Lyotard y Derrida. La crisis en la enseñanza de las humanidades ha sido detectada denunciada, descrita y analizada hace ya bastante tiempo. En este sentido la investigación de Nussbaum no es una novedad. Sílo es, en cambio, su abordaje metodológico y propositivo. En cuanto a lo primero usa como herramienta conceptual la filosofía y la teoría de la educación para establecer una relación de causa-efecto de las políticas económicas globales sobre la educación. En cuanto al segundo, muestra la "pernicia" del actual modelo educativo global frente a la formación para la democracia y propone uno nuevo que, centrado en la enseñanza de las humanidades, y a través del "juego" como estrategia pedagógica, abogue por el "desarrollo humano" como elemento fundamental para la construcción de un civismo de connotaciones mundiales. ${ }^{3}$ En ambos abordajes Nussbaum no se queda en la pura reflexión filosófica sino que sustenta cada tesis y cada

1 NUSSBAUM, Martha. Sin fines de lucro: por qué la democracia necesita de las humanidades. Trad. María Victoria Rodil. Madrid: Katz Editores, 2010. p. 19-31.

Martha Nussbaum (1947-) es una filósofa estadounidense cuyos intereses investigativos en las universidades de Harvard, Brown y Chicago, entre otras, se centran en la filosofía antigua, la filosofía política, la filosofía del derecho y las humanidades. Sobre este último tópico ha escrito libros como El ocultamiento de lo humano: repugnancia, vergüenza y ley (2006).

2 Véase, FOUCAULT, Michel. "El sujeto y el poder". En: Revista de Ciencias Sociales. Montevideo: Fundación de Cultura Universitaria, No. 1.. 1996. p. 3- 4.

3 NUSSBAUM. M. Op. cit. p. 28-30. 
argumento ejemplificando con hechos. Sócrates, Tagore, Dewey y Rousseau, son algunas de las fuentes teóricas de las que se vale Nussbaum para argumentar su planteamiento central: la enseñanza de las humanidades es necesaria para el futuro de la democracia en términos de su desarrollo cívico. ${ }^{4}$

En coherencia con lo hasta aquí expuesto, el presente artículo tiene en la investigación de Nussbaum una de sus principales herramientas teóricas para llevar a cabo lo que se propone: describir y analizar, desde una perspectiva genealógica, la relación histórica entre universidad y humanidades en sus aspectos político y pedagógico, como se verá, estrechamente relacionados. La genealogía es una herramienta metodológica para describir y analizar la historia. Su punto de partida es un problema en el presente, el cual intenta deconstruir para mirar cómo emergió en el pasado dicho problema. No se trata, como en la historia tradicional, de una enumeración cronológica de fechas, hitos y nombres de héroes, sino de la descripción y análisis de las condiciones sociales de posibilidad de luchas y conflictos en que un problema determinado emerge. "Las fuerzas presentes en la historia no obedecen ni a un destino ni a una mecánica, sino al azar de la lucha". ${ }^{5}$

Por humanidades entendemos un saber sobre lo humano, el cual tendría tres dimensiones principales: las disciplinas del saber sobre el ser humano, la crítica, creatividad y ética, esto es, "la búsqueda del pensamiento crítico y los desafíos a la imaginación, así como con la comprensión empática de una variedad de experiencias humanas y de la complejidad que caracteriza a nuestro mundo". ${ }^{6}$ Esto a partir de un intento de respuesta a la pregunta por las condiciones sociales de posibilidad en que las humanidades son enseñadas actualmente en la universidad.

Para tal efecto, partimos de la siguiente hipótesis: en la universidad de hoy las humanidades son enseñadas en condiciones de saber y de poder. Estas condiciones sin embargo, no emergen durante el presente siglo sino en el siglo XVIII con la aparición de la universidad burguesa europea, haciéndose discursivamente visibles a finales de este mismo siglo con la publicación del libro El conflicto de las facultades de Immanuel Kant.

${ }^{4}$ Ibid. p. 26.

5 FOUCAULT, Michel. "Nietzsche, la genealogía y la historia". En: Microfísica del poder. Madrid: La Piqueta 1980. p. 20.

6 NUSSBAUM. M. Op. cit.p. 26. 


\section{Educación y democracia: panorama actual de la enseñanza de las humanidades}

En términos de su impacto sobre valores fundamentales de la democracia, como lo son la libertad y la justicia, y sobre la democracia misma en tanto que forma de gobierno, la crisis económica global que se desató a comienzos del año 2008 podría llegar a ser "menos perjudicial" que la actual crisis educativa en lo que respecta a la enseñanza de las humanidades. Esta crisis, que se expresa en los "cambios drásticos" que se están produciendo en los sistemas educativos de los estados democráticos con respecto a la enseñanza de los saberes, tiene su origen en las políticas educativas estatales que ven en el fomento de las carreras técnicas y tecnológicas, así como en la promoción de carreras profesionales tradicionales directamente relacionadas con actividades empresariales, una estrategia para asegurar su competitividad económica en el mercado mundial. Es claro que esta estrategia está determinando las tendencias curriculares contemporáneas y con ello la erradicación de saberes específicos como las humanidades, según los tecnócratas del currículo, nada útiles en estos tiempos de globalización. "Si esta tendencia se prolonga", vaticina Nussbaum, "las naciones de todo el mundo en breve producirán generaciones enteras de máquinas utilitarias, en lugar de ciudadanos cabales con la capacidad de pensar por sí mismos, poseer una mirada crítica sobre las tradiciones y comprender la importancia de los logros y los sufrimientos ajenos. El futuro de la democracia a escala mundial pende de un hilo". ${ }^{7}$

Lo anterior, en términos generales, constituye el panorama actual de la enseñanza de las humanidades en el mundo desde el punto de vista de Martha Nussbaum. Al respecto tenemos dos inquietudes: ¿Por qué, en lo que tiene que ver con su impacto sobre la democracia, la crisis económica que comenzó en el año 2008 podría llegar a ser "menos perjudicial" que la actual crisis educativa en lo que respecta a la enseñanza de las humanidades? ¿Cuáles son los hechos que sustentan la afirmación de que la actual crisis educativa, en lo atinente a la enseñanza de las humanidades, es de connotación mundial?

En efecto, la economía es el sustento no solo de la democracia sino de toda forma de gobierno. Para que la democracia sea sostenible en el tiempo es necesario una organización económica que dinamice y haga competitiva la

7 NUSSBAUM. M. Op. cit. p. 20.

Universidad y humanidades - p. 97 - 112 
producción de bienes y servicios de un Estado de cara al mercado mundial. Esto es innegable. Sin embargo, este es apenas el sustento material de la democracia. El otro es el sustento cívico que, a través de la enseñanza de las humanidades, le proporciona la educación. En este sentido, aunque es evidente que hasta la educación misma requiere de la organización económica para su funcionamiento, el impacto que la actual crisis mundial educativa tiene sobre la democracia podría llegar a ser de proporciones dantescas frente a la crisis económica en la medida en que esta última es "ruidosa", "publicitada" y, en efecto, atendida de manera urgente por los gobiernos por medio de medidas económicas extraordinarias de corto y largo plazo, en cambio, la crisis educativa, específicamente la crisis por la que atraviesa la enseñanza de las humanidades, es "silenciosa" y pasa casi inadvertida tanto por los gobiernos como por los ciudadanos, e incluso, por los directamente involucrados, maestros y estudiantes: "La crisis nos mira de frente, pero aún no la hemos enfrentado. Continuamos como si todo siguiera igual que siempre, cuando en realidad resulta evidente en todas partes que ya no se pone el acento en lo mismo que antes". 8

Son varios los hechos que sustentan la afirmación de Nussbaum de que la actual crisis en materia de educación, en lo que respecta a la enseñanza de las humanidades, es de connotación mundial. Los contextos en los que se desarrollan tales hechos son básicamente de índole investigativo, filosófico y gremial. En cuanto al primer contexto, Nussbaum pone como ejemplo el informe del año 2006 sobre el estado de la educación en la universidad norteamericana, titulado El liderazgo a prueba: un mapa del futuro de la educación superior en los Estados Unidos, coordinado por la entonces secretaria de educación del gobierno del presidente George Bush: "Este informe presentaba una crítica significativa contra la desigualdad en el acceso a la educación superior. No obstante, en términos de contenidos, se centra por completo en la educación para el beneficio económico nacional. El texto apuntaba a las deficiencias en materia de ingeniería, ciencia y tecnología, mas no a la investigación científica en esos campos, sino al aprendizaje del conocimiento aplicado, que sirve para generar velozmente estrategias destinadas a la obtención de renta". ${ }^{9}$ En cuanto al segundo contexto, Nussbaum apela al caso de India. Como se sabe, desde finales del siglo XIX y hasta mediados del siglo XX, este país tuvo una fuerte influencia sobre los Estados Unidos,

8 Ibid. p. 21.

9 Ibid. p. 21-22. 
Japón y Europa en lo que atañe a la filosofía de la educación, la cual estaba centrada en la experiencia pedagógica de Rabindranath Tagore, consistente en la "atribución de poder social a sus alumnos mediante la práctica del método socrático, la exposición a diferentes culturas y, sobre todo, la inclusión de la música, las bellas artes, el teatro y la danza en todas las secciones del programa curricular". ${ }^{10}$ Lo que llama la atención de Nussbaum, es que "Hoy en día, las ideas de Tagore son desestimadas e incluso desdeñadas en la India" "11 dadas las políticas globales de rentabilidad de las que se han contagiado sus gobiernos y que ha desplazado "el desarrollo humano basado en la imaginación y en el pensamiento crítico con la que Tagore había formado a los futuros ciudadanos de la democracia exitosa que logró dicho país". ${ }^{12}$ Por último, y en cuanto al contexto gremial, Nussbaum trae a colación el hecho de que los maestros y directivos de la universidad norteamericana tienen poco interés investigativo en la enseñanza de las humanidades y su importancia "en la educación para la ciudadanía democrática". ${ }^{13}$

Acerca de todo lo anterior creemos que, si bien es cierto la muestra poblacional (Estados Unidos e India) que Nussbaum utiliza para mostrar el grado de abandono en el que se encuentra la enseñanza de las humanidades en el mundo es insuficiente, también es cierto que los países que constituyen dicha muestra son históricamente representativos en lo que respecta a reformas educativas y experiencias pedagógicas de influencia mundial, especialmente para Europa en el caso de india y para Latinoamérica en el caso de Estados Unidos. ${ }^{14}$ En este sentido consideramos que las afirmaciones de Nussbaum no solo son válidas sino que actúan como argumentos a favor de su planteamiento central (la enseñanza de las humanidades es necesaria para el futuro de la democracia en términos de su desarrollo cívico), pues evidentemente una democracia sin sustento cívico, aunque prospera económicamente, es una democracia incompleta.

Ahora bien, en rigor la descripción y análisis que hace Nussbaum del actual panorama de la enseñanza de las humanidades en el mundo, tanto a nivel de la

${ }^{10}$ Ibid.

${ }^{11}$ Ibid.

${ }^{12}$ Ibid.

${ }^{13}$ Ibid.

${ }^{14}$ Para profundizar en la influencia de las políticas educativas de los Estados Unidos en Latinoamérica véase, UNESCO, La situación educativa en América Latina, Washington, Unesco, 1960. 
educación primaria, secundaria y universitaria, es a nuestro juicio una investigación sobre las condiciones de posibilidad en que hoy se enseñan las humanidades. Dichas condiciones son fundamentalmente dos: el saber (se privilegia una "educación para la renta" en detrimento de una "educación para la democracia") ${ }^{15}$ y el poder (en el contexto del actual paradigma mundial de "crecimiento económico", los estados democráticos encuentran en la educación la estrategia propicia para determinar lo que deben saber (aprender) los sujetos de acuerdo con los intereses productivos de sus gobiernos) ${ }^{16}$. Así pues, el tipo de saber que aprenden los sujetos en las instituciones educativas (especialmente en la universidad) está determinado por una estrategia de poder de procedencia estatal, la cual supone un tipo de saber, en este caso, el saber del gobierno sobre lo que considera deben saber sus ciudadanos en coherencia con su modelo económico de desarrollo cada vez más desligado de la formación humanística. En este sentido el currículo se convierte en un campo de saber y de poder: "Seleccionar es una operación de poder. Privilegiar un tipo de conocimiento es una operación de poder. Destacar, entre las múltiples posibilidades, una identidad o subjetividad como ideal es una operación de poder". ${ }^{17}$ De ahí nuestra hipótesis: en la universidad de hoy las humanidades son enseñadas en condiciones de saber y de poder.

\section{Saber y poder: panorama histórico de la enseñanza de las humanidades en la Universidad}

Contrario a lo que comúnmente se cree, el preocupante panorama de la enseñanza de las humanidades descrito por Nussbaum no sólo es actual sino histórico. Ya en los años setenta del siglo XX, Jean François Lyotard, ${ }^{18}$ llamaba la atención acerca de la determinante influencia de las políticas económicas globales sobre el saber en la educación. Tal influencia es conocida en Lyotard como La condición postmoderna. ${ }^{19}$ Se trata de un informe sobre cómo, por

15 Ibid. p. 33-48

16 Ibid.

17 TADEU DA SILVA, Tomaz. Espacios de identidad: nuevas visiones sobre el currículum. Trad. Estanislao Antelo. Brasil:.Octaedro. 2001. p. 17.

${ }^{18}$ LYOTAYD, Jean François. La condición postmoderna: informe sobre el saber. Trad. Mariano Antolín Rato. Madrid: Ed. Cátedra. 1989.

${ }^{19}$ En Lyotard el término postmodernidad se entiende como "el estado de la cultura después de las transformaciones que han afectado a las reglas de juego de la ciencia, de la literatura y de las artes a partir del siglo XIX”. Véase, Lyotard. Ibid. p. 4. 
efecto de las trasformaciones técnicas, tecnológicas, y en general científicas, que se han producido en el mundo partir de la segunda postguerra (las cuales deben su aparición a una política de globalización de la economía que ve en los mercados internos de los Estados democráticos simples piezas productivas de un mercado único internacional), el saber ha cambiado de "estatuto" alterando con ello la dinámica misma de la educación. Lyotard muestra que, en términos del saber, en la postmodernidad se produce una ruptura con respecto a la modernidad. Dicha ruptura se expresa en la manera como en cada una de estas épocas se concibe el saber, pues, mientras en la modernidad el saber se concibe como un factor fundamental en la búsqueda de la libertad y la igualdad de todos los hombres, en la postmodernidad se concibe como un factor sólo útil en los procesos de producción de los Estados.

Así pues, aunque Lyotard no hace alusión directa a las humanidades y su enseñanza, a partir de la ruptura que sobre el saber describe, se pude inferir que aquel estatuto humanístico que tuvo el saber en la modernidad, y que aparece directamente relacionado con los valores de libertad y justicia, es cooptado en la postmodernidad por los intereses económicos multinacionales que le dan al saber el tratamiento de mera "información", precipitando de este modo el fracaso de la modernidad en lo relacionado con lo que podríamos llamar su proyecto humanístico, en el que el saber se deriva de la razón en tanto que facultad propia del hombre y por medio de la cual cada individuo se interesaría no sólo por comprender sino por comunicar autónomamente las dimensiones fundamentales que lo constituyen como sujeto: la historia y la ciencia. La compresión de la historia y de la ciencia sería el principal objetivo de la educación moderna en la transmisión del saber. Objetivo también cooptado en la postmodernidad en la medida en que, dada la investidura de mercancía que adquiere el saber, las instituciones educativas cada vez más se ven avocadas a jugar un papel empresarial, es decir, de administradoras del saber de acuerdo con los lineamientos curriculares determinados por los tecnócratas de los Ministerios de Educación Nacional en relación con las políticas educativas estatales a su vez ajustadas a las políticas económicas globales de rentabilidad. ${ }^{20}$

Ahora bien, lo problemático de la cuestión anterior, como el mismo Lyotard lo señala, es que hoy más que en otra época el saber es un asunto de poder: la

${ }^{20}$ Para profundizar en la relación, políticas económicas, educación, historia, ciencia y transmisión del saber a partir del siglo XIX, véase, Lyotard, "Pragmática del saber narrativo". En: Ibid., cap. 6, p. 43-44. 
"cuestión del saber en la edad de la informática es más que nunca la cuestión del gobierno". ${ }^{21}$ Esto se explica en el hecho de que hoy el hombre no se interesa por adquirir un saber, como en la modernidad, porque dicho saber lo hará libre, autónomo, justo, creativo, solidario, crítico, es decir, humano, sino porque ese saber, en el contexto del capitalismo actual, le da la posibilidad de ocupar un sitio estratégico de poder. Tal es el caso de los gobernantes de los Estados democráticos, quienes para tales efectos se interesan en el saber político como sitio estratégico de poder, desde el cual coordinan la producción del saber en tanto que información y deciden acerca de los mecanismos más apropiados para su difusión.

Esta cuestión, la del saber y su relación con el poder, siguiendo los planteamientos de Nussbaum, hoy afecta la enseñanza de las humanidades en la medida en que los gobernantes, urgidos de acciones para incrementar la producción interna en sus países con el objeto de permanecer competitivos en el mercado mundial, tienden cada vez más a privilegiar la enseñanza de saberes técnico-administrativos en detrimento de la enseñanza de las humanidades.

La historia nos muestra que este problema, el de la relación saber-poder y sus efectos sobre la enseñanza de las humanidades, tiene su antecedente más próximo en el siglo XVIII, con la aparición de la universidad burguesa. Esta universidad, resultado del ascenso del poder político de la burguesía frente a la monarquía, hace entrar en crisis el modelo universitario medieval cuyo saber responde a los requerimientos del mundo agrícola-feudal salvaguardado y promovido por los gremios o sociedades de académicos cerradas:

Al lado de la acostumbrada renta territorial, aparece y se consolida rápidamente la ganancia industrial vinculada a la expansión y al empleo racional del capital comercial y financiero, lo cual implica la transformación y el mejoramiento de las técnicas de producción, la necesidad de un uso diverso y diferenciado de la fuerza de trabajo, así como la creación de nuevas condiciones para la vida individual y social. Así, decaen hasta su desaparición, las viejas estructuras de la sociedad posterior al Medievo. Los privilegios feudales de la nobleza, las intrincadas reglas de los antiguos gremios, los lentos aparatos administrativos se manifiestan insuficientes y obsoletos respecto a las nuevas necesidades que se perfilan. Todo esto entrará en una rápida disolución. ${ }^{22}$

${ }^{21}$ Ibid. p. 24.

${ }^{22}$ BONVECCHIO, Claudio. El mito de la universidad. Trad. María Esther Aguirre Lora. Siglo XXI editores. México. 1991. P. 27-28. 
En este escenario de ejercicio del poder político en el que la burguesía (la renta industrial y financiera) se impone a la monarquía (la renta feudal o territorial), el saber se transforma de instrumento de poder de la producción agrícolafeudal a instrumento de poder de la producción técnica, industrial y financiera de la burguesía. “...en el saber es donde la burguesía cree poder encontrar las modalidades funcionales ya sea para su propia reproducción y selección interna, ya para el propio control que ejercerá sobre el orden social". ${ }^{23}$ La universidad se erige así en el lugar privilegiado de este saber y del poder que en él se inscribe. Así lo sugiere Bonvecchio cuando escribe:

"Tal vez por primera vez en la historia, el saber parece asumir como finalidad propia aquella dimensión que desde siempre había sido teorizada a partir de Platón, pero que jamás se había poseído en lo concreto: la coincidencia entre el saber y el poder. En las figuras de los iluministas europeos (franceses, alemanes, ingleses) parece encarnarse el filósofo-rey: el poder asume las connotaciones del saber, y el saber las del poder. Los grandes reformadores son eruditos o filósofos, mientras que los grandes proyectos de transformación social ya no se elaboran en los salones de las cortes europeas, sino en las bibliotecas y en la academia [...]. El objeto prioritario de los últimos años del siglo XVIII y de los primeros del siglo XIX, es la educación universitaria: la universidad debe llagar a ser el símbolo material de la trama que el saber está tejiendo, o cree tejer, en la sociedad". ${ }^{24}$

No obstante, este cuadro histórico en el que el saber y el poder se relacionan en el ámbito universitario, no surge de manera intempestiva sino bajo el influjo de ciertas condiciones de organización provenientes del Estado: reestructuración racional de las facultades, establecimiento de normas rigurosas para la admisión de los estudiantes, actualización de los programas académicos, etc. En este sentido, en lo concerniente al siglo XIX, es relevante la influencia de la filosofía hegeliana fundamentalmente en dos nociones: Estado y espíritu absoluto. La primera es la ética universal institucionalizada. La segunda es el arte, la religión y la filosofía, que en rigor son tres formas como el hombre se representa así mismo y a sus criterios. ${ }^{25}$

Aunque efectivamente la influencia del pensamiento hegeliano ha sido determinante en la distribución del saber y del poder en la universidad del

${ }^{23}$ Ibid. p. 29.

${ }^{24}$ Ibid. p 29-30.

${ }^{25}$ Véase. BONVECCIO. Ibid. p. 30. 
siglo XIX, creemos que el problema de la relación del saber con el poder en la universidad adquiere plena visibilidad discursiva desde finales del siglo XVIII con la publicación de El conflicto de las facultades (1789) de Immanuel Kant. Aunque Kant, al igual que Lyotard, no habla de la enseñanza de las humanidades propiamente dicha, sí, en cambio, evidencia la relación histórica entre universidad y humanidades en términos de saber y de poder al considerar conflictiva la relación de las facultades (Facultades superiores: teología, derecho y medicina vs facultad inferior: filosofía) al interior de la universidad. "La disputa de las facultades -escribe Kant- gira en torno a su incidencia en el I pueblo y sólo pueden conseguir dicha influencia por cuanto cada una de ellas haga creer al pueblo que conoce el mejor modo para fomentar su dicha, aunque sean tan opuestos el uno del otro". ${ }^{26}$

En este sentido, Kant muestra que la pugna entre dichas facultades y su división misma, no obedece exclusivamente a una cuestión de saber, y especialmente de saber científico, sino que tiene su fundamento-origen en el poder gubernamental que atraviesa estructuralmente a la universidad:

Conforme al uso establecido se dividen en dos categorías: tres Facultades superiores y una Facultad inferior. Como es obvio, para esta división y su correspondiente nomenclatura, no se ha consultado al mundo académico, sino al gobierno. Pues entre las superiores sólo se encuentran aquellas sobre cuyas doctrinas le interesa al gobierno determinar cuáles hayan de ser sus contenidos o si deben ser expuestos públicamente; por el contrario, aquella que sólo ha $\backslash$ de velar por el interés de la ciencia, es llamada inferior, dado que ésta puede tratar sus tesis a su antojo. Al gobierno le interesa por encima de todo aquello que procura un fuerte y duradero influjo sobre el pueblo, y de esa índole son las materias de las facultades superiores. ${ }^{27}$

Así pues, aunque Kant, en términos conceptuales, no analiza estrictamente la relación del saber con el poder en el ámbito universitario, de dicho análisis se puede deducir tal relación con respecto a la enseñanza de las humanidades en la medida en que evidencia un conflicto de saber-poder entre las facultades en mención, el cual trata de dirimir cuando dice:

${ }^{26}$ KANT, Immanuel. El conflicto de las facultades. Alianza Editorial. Trad. Roberto R. Aramayo. Madrid. 2003. p. 58.

${ }^{27}$ Ibid. p. 64. 
La facultad de filosofía comprende dos departamentos, el de la ciencia histórica (donde se inscriben la historia, la geografía, la filología, las humanidades con todo cuanto presenta la ciencia natural del conocimiento empírico) y el de las ciencias racionales puras (matemática pura y de la filosofía pura, metafísica de la naturaleza y de las costumbres), así como la mutua correlación entre ambas partes del saber. Abarca, pues, todos los ámbitos del conocimiento humano (y, por ende, desde un punto de vista histórico también las facultades superiores) constituyen contenidos suyos, sino objetos de su examen y crítica en aras del provecho de las ciencias. ${ }^{28}$

Por todas las razones anteriores es que consideramos que con la publicación de El conflicto de las facultades de Emmanuel Kant, el saber y el poder, como condiciones de posibilidad de la enseñanza de las humanidades en la universidad, se hacen discusivamente visibles.

\section{La enseñanza de las humanidades en la universidad, un objeto histórico de saber y de poder. (A modo de conclusión)}

De acuerdo con la descripción y análisis que hemos hecho acerca de los planteamientos de Nussbaum, Lyotard, Bonvecchio y Kant, con respecto a la relación universidad y humanidades, podemos concluir que la enseñanza de las humanidades en la universidad constituye un objeto histórico de saber y de poder. Esto quiere decir, de un parte, que el saber y el poder han sido históricamente condiciones de posibilidad de dicha enseñanza en el ámbito universitario (el aspecto político de la cuestión: el poder gubernamental decidiendo lo que se debe enseñar y aprender en las instituciones educativas de acuerdo con el paradigma de cada época), y, de otra parte, que en el sentido histórico referido, el saber y el poder han atravesado la enseñanza de las humanidades propiamente dicha (el aspecto pedagógico de la cuestión: qué enseñar a nivel de contenidos y cómo hacerlo).

En este último sentido ayer y hoy el trabajo recae sobre el maestro. Dicho trabajo es complejo pero posible. Para tal efecto se requiere de un nuevo maestro universitario de humanidades, que resignifique en la teoría y en la práctica a la universidad misma en relación con la sociedad, esto es, un maestro en constante actividad crítica, creativa y ética frente a problemas de fondo como la autonomía universitaria y las políticas educativas estatales, la libertad de

${ }^{28}$ Ibid. p. 77.

Universidad y humanidades - p. 97 - 112 
cátedra y la investigación, el origen y sentido de la academia, etc, es decir, un maestro verdaderamente humanística, comprometido no solo a nivel curricular con los contenidos y temáticas históricas (lenguaje, arte, sociedad, etc. ) sino con su entorno social, político, cultural y económico, que comunique el presente con el pasado histórico (un maestro genealogista diría Nietszche), que influencie a sus estudiantes con su creatividad.

Sin embargo, y dado que desde hace tiempo "La universidad como el gran templo laico de la cultura quedó disuelta, ya que, efectivamente, en la actualidad resulta incompatible con la función tecnológica-burocrática que el modelo de reproducción social le atribuye en el capitalismo avanzado", ${ }^{29}$ además de un nuevo maestro de humanidades, la universidad de hoy requiere, para la revitalización del espíritu científico que la caracterizó en su génesis, un nuevo maestro universitario, como lo reclamara el italiano Carlo Cantoni ${ }^{30}$ en el siglo XIX, un maestro científicohumanista, y con él una nueva universidad, como la caracteriza Jacques Derrida, ${ }^{31}$ "sin condición", que no sea sólo titulante sino investigadora, conceptualmente deconstructiva, con compromiso social, y en la que unas "nuevas humanidades" sean el principio mismo de dicha incondicionalidad:

Intentaré precisar lo que entiendo por «nuevas» Humanidades. Pero, ya sean estas discusiones críticas o deconstructivas, lo que concierne a la cuestión y a la historia de la verdad en su relación con la cuestión del hombre, de lo propio del hombre, del derecho del hombre, del crimen contra la humanidad, etc., todo ello debe en principio hallar su lugar de discusión incondicional y sin presupuesto alguno, su espacio legítimo de trabajo y de reelaboración, en la universidad y, dentro de ella, con especial relevancia, en las Humanidades. No para encerrarse dentro de ellas sino, por el contrario, para encontrar el mejor acceso a un nuevo espacio público transformado por unas nuevas técnicas de comunicación, de información, de archivación y de producción de saber. ${ }^{32}$

En este orden de ideas, pero en lo que respecta al problema de la enseñanza de las humanidades en cuanto al qué enseñar y cómo enseñar, el profesor Guillermo

${ }^{29}$ BONVECCHIO. Op. cit. p. 21.

${ }^{30}$ CANTONI, Carlo. "Los defectos de los estudiantes y de los maestros". En: El mito de la Universidad. BONVECCHIO, Claudio. Op. Cit. p. 175-186.

${ }^{31}$ DERRIDA, Jacques. La universidad sin condición. Trad. Cristina Peretti y Paco Vidarte. Edición digital de Derrida en castellano. http://www.jacquesderrida.com.ar/textos/ universidad-sin-condicion.htm.

${ }^{32}$ Ibid. p. 2. 
Hoyos Vásquez plantea "la posibilidad de una educación para un nuevo humanismo en términos de la dialéctica trascendental de Kant: es decir como reconocimiento, en los límites, de la experiencia mundo vital y del conocimiento científico, dejando abierta la posibilidad del pensar en dichos límites, como complementariedad necesaria de la moral y de la dimensión estética". ${ }^{33}$ Esto implica, por un lado "reexaminar" la educación empresa que desprecia la educación en valores y por ende es indiferente a la enseñanza de las humanidades, y por otro lado, recontextualizar el concepto mismo de humanidades, pues como a renglón seguido escribe Hoyos:

La situación hoy es la de tal avance en el conocimiento científico en áreas como la biogenética, la robótica y las neurociencias, que no parece racional ignorar el influjo que tales saberes adquieren con respecto a la autocomprensión de las personas y a los discursos públicos en torno al sentido mismo de la libertad y de las capacidades de autodeterminación y responsabilidad de las personas. ${ }^{34}$

En conclusión, en el presente artículo, y desde una perspectiva genealógica, hemos descrito y analizado, las relaciones del saber con el poder en el ámbito universitario histórico, específicamente entorno a la enseñanza de las humanidades, significando con ello, de una parte, que tal enseñanza ha sido histórica y sistemáticamente menospreciada a nivel curricular y a nivel cultural del campo académico universitario como consecuencia de una estrategia de poder gubernamental que ha tenido distintos intereses sobre el saber en distintas épocas, y de otra parte, dado el ejercicio de poder sobre el saber, que la enseñanza de las humanidades ayer y hoy no tienen como único centro problemático la cuestión pedagógica en tanto que contenidos temáticos y estrategias metodológicas, sino que es un problema estructural que tiene que ver tanto con el aspecto político como el pedagógico. En este sentido urge no sólo un nuevo maestro de humanidades sino un maestro universitario científicohumanista, unas "nuevas humanidades", en suma, una universidad "sin condición".

${ }^{33}$ HOYOS VASQUEZ, Guillermo. "Educación para un nuevo humanismo en tiempos de globalización”. En: Revista Cuestiones de filosofía”. No. 12. U.P.T.C. Tunja, 2010.

34 Ibid. 


\section{Bibliografía}

BOVECCHIO, Claudio. El mito de la universidad. México. Siglo XXI editore. 1991.

DERRIDA, Jacques. La universidad sin condición. Edición digital de Derrida en castellano. http://www.jacquesderrida.com.ar/textos/universidad-sincondicion.htm.

HOYOS VASQUEZ, Guillermo. "Educación para un nuevo humanismo en tiempos de globalización". En: Revista Cuestiones de filosofía". No. 12. UPTC. Tunja, 2010.

FOUCAULT, Michel. "El sujeto y el poder". En: Revista de Ciencias Sociales. Fundación de Cultura Universitaria. No. 1. Montevideo. 1996.

FOUCAULT, Michel. "Nietzsche, la genealogía y la historia". En: Microfísica del poder. La piqueta. Madrid. 1980.

KANT, Emmanuel. El conflicto de las facultades. Madrid. Alianza Editorial. 2003.

LYOTAYD, Jean François. La condición postmoderna: informe sobre el saber. Madrid. Ed. Cátedra. 1989.

NUSSBAUM, Martha. Sin fines de lucro: por qué la democracia necesita de las humanidades. Madrid. Katz Editores. 2010.

TADEU DA SILVA, Tomaz. Espacios de identidad: nuevas visiones sobre el currículum. Brasil. Octaedro. 2001.

UNESCO, La situación educativa en América Latina. Washington, Unesco, 1960. 